\title{
Sensitivity of the High Altitude Water Cherenkov Detector to Sources of Multi-TeV Gamma Rays
}

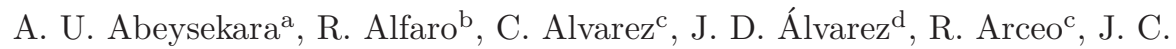 \\ Arteaga-Velázquez ${ }^{\text {d }}$ H. A. Ayala Solares ${ }^{\mathrm{e}}$, A. S. Barberf ${ }^{\mathrm{f}}$ B. M. Baughman ${ }^{\mathrm{g}, * *}$, \\ N. Bautista-Elivarh ${ }^{\text {, E. Belmont }}{ }^{\mathrm{b}}$, S. Y. BenZvi ${ }^{\mathrm{i}}$, D. Berley ${ }^{\mathrm{g}}$, M. Bonilla \\ Rosales $^{\mathrm{j}}$, J. Braun ${ }^{\mathrm{g}}$, R. A. Caballero-Lopez ${ }^{\mathrm{k}}$, A. Carramiñana ${ }^{\mathrm{j}}$, M. Castillol, \\ U. Cotti ${ }^{\mathrm{d}}$, J. Cotzomi ${ }^{1}$, E. de la Fuente ${ }^{\mathrm{m}}$, C. De León ${ }^{\mathrm{d}}$, T. DeYoung ${ }^{\mathrm{n}}$, R. Diaz \\ Hernandez ${ }^{j}$, J. C. Diaz-Velez ${ }^{i}$ B. L. Dingus ${ }^{\circ}$, M. A. DuVernoisis , R. W. \\ Ellsworth $^{\mathrm{p}, \mathrm{g}}$, A. Fernandez , D. W. Fiorino ${ }^{\mathrm{i}}$, N. Fraija ${ }^{\mathrm{q}}$, A. Galindo ${ }^{\mathrm{j}}$, J. L. \\ Garcia-Luna $^{\mathrm{m}}$, G. Garcia-Torales ${ }^{\mathrm{m}}$, F. Garfias ${ }^{\mathrm{q}}$, L. X. González ${ }^{\mathrm{k}}$, M. M. \\ González $^{\mathrm{q}}$, J. A. Goodman ${ }^{\mathrm{g}}$, V. Grabski ${ }^{\mathrm{b}}$, M. Gussert ${ }^{\mathrm{r}}$, Z. Hampel-Arias ${ }^{\mathrm{i}}$, C.

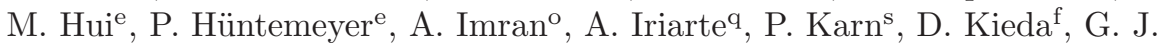 \\ Kunde $^{\mathrm{o}}$, A. Lara ${ }^{\mathrm{k}}$, R. J. Lauer ${ }^{\mathrm{t}}$, W. H. Lee ${ }^{\mathrm{q}}$, D. Lennarz ${ }^{\mathrm{u}}$, H. León Vargas ${ }^{\mathrm{b}}$, \\ E. C. Linares ${ }^{\mathrm{d}}$, J. T. Linnemann ${ }^{\mathrm{a}}$, M. Longo ${ }^{\mathrm{r}}$, R. Luna-Garcíav ${ }^{\mathrm{v}}$, A. Marinelli ${ }^{\mathrm{b}}$, \\ O. Martinez ${ }^{1}$, J. Martínez-Castrov, J. A. J. Matthews ${ }^{\mathrm{t}}$, P. \\ Miranda-Romagnoliw,j, E. Moreno ${ }^{1}$, M. Mostafár ${ }^{\mathrm{r}}$, J. Nava ${ }^{\mathrm{j}}$, L. Nellen ${ }^{\mathrm{x}}, \mathrm{M}$. \\ Newbold $^{f}$, R. Noriega-Papaqui ${ }^{w}$, T. Oceguera-Becerra ${ }^{\mathrm{m}, \mathrm{b}}$, B. Patricelliq, R. \\ Pelayo $^{1}$, E. G. Pérez-Pérez ${ }^{\mathrm{h}}$, J. Pretz ${ }^{\mathrm{o}, *}$, C. Rivière ${ }^{\mathrm{q}}$, D. Rosa-González ${ }^{\mathrm{j}}, \mathrm{H}$.

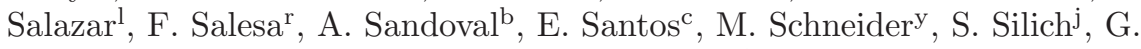

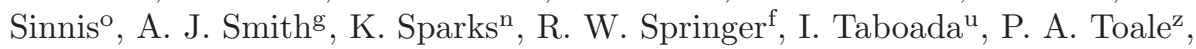 \\ K. Tollefson ${ }^{\mathrm{a}}$, I. Torres ${ }^{\mathrm{j}}$, T. N. Ukwatta ${ }^{\mathrm{a}}$, L. Villaseñor ${ }^{\mathrm{d}}$, T. Weisgarber ${ }^{\mathrm{i}}, \mathrm{S}$.

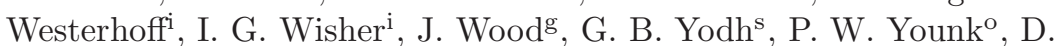 \\ Zaborov $^{\text {n }}$ A. Zepeda ${ }^{\text {aa }}$, H. Zhou ${ }^{\text {e }}$

\footnotetext{
${ }^{a}$ Department of Physics and Astronomy, Michigan State University, East Lansing, MI, USA

${ }^{b}$ Instituto de Física, Universidad Nacional Autónoma de México, Mexico D.F., Mexico ${ }^{c}$ CEFyMAP, Universidad Autónoma de Chiapas, Tuxtla Gutiérrez, Chiapas, Mexico ${ }^{d}$ Universidad Michoacana de San Nicolás de Hidalgo, Morelia, Mexico ${ }^{e}$ Department of Physics, Michigan Technological University, Houghton, MI, USA ${ }^{f}$ Department of Physics and Astronomy, University of Utah, Salt Lake City, UT, USA ${ }^{g}$ Department of Physics, University of Maryland, College Park, MD, USA ${ }^{h}$ Universidad Politecnica de Pachuca, Pachuca, Hgo, Mexico ${ }^{i}$ Department of Physics, University of Wisconsin-Madison, Madison, WI, USA ${ }^{j}$ Instituto Nacional de Astrofísica, Óptica y Electrónica, Puebla, Mexico

${ }^{k}$ Instituto de Geofísica, Universidad Nacional Autónoma de México, Mexico D.F., Mexico

${ }^{l}$ Facultad de Ciencias Físico Matemáticas, Benemérita Universidad Autónoma de Puebla, Puebla, Mexico

${ }^{m}$ Dept. de Fisica; Dept. de Electronica (CUCEI), IT.Phd (CUCEA), Phys. Mat. Phd (CUVALLES), Universidad de Guadalajara, Jalisco, Mexico

${ }^{n}$ Department of Physics, Pennsylvania State University, University Park, PA, USA ${ }^{\circ}$ Physics Division, Los Alamos National Laboratory, Los Alamos, NM, USA ${ }^{p}$ School of Physics, Astronomy, and Computational Sciences, George Mason University, Fairfax, VA, USA
} \\ ${ }^{q}$ Instituto de Astronomía, Universidad Nacional Autónoma de México, Mexico D.F., Mexico
}

*jpretz@lanl.gov
**bbaugh@umdgrb.umd.edu 


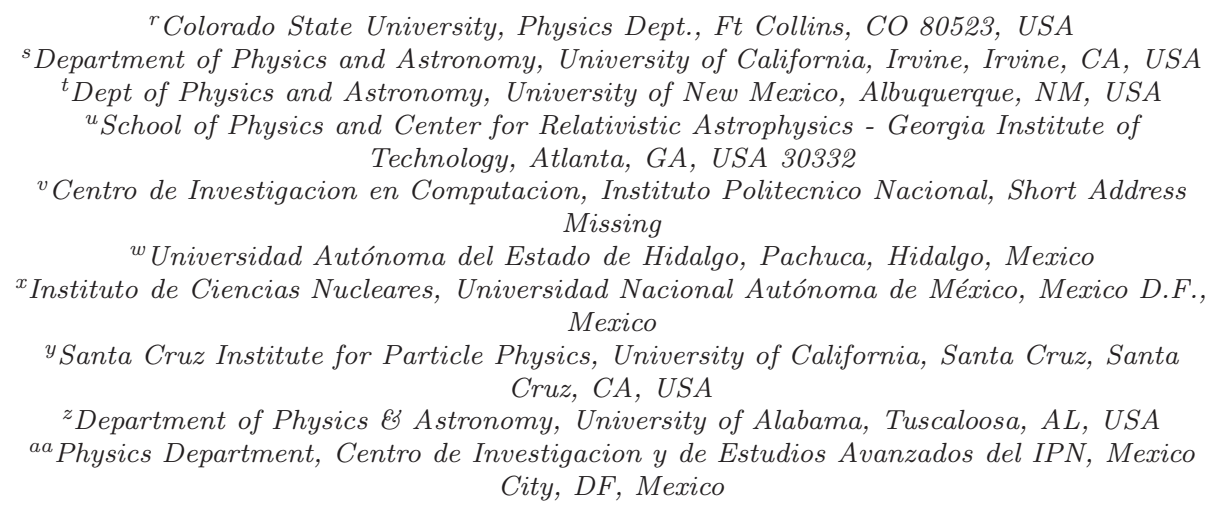

\begin{abstract}
The High Altitude Water Cherenkov (HAWC) observatory is an array of large water Cherenkov detectors sensitive to gamma rays and hadronic cosmic rays in the energy band between $100 \mathrm{GeV}$ and $100 \mathrm{TeV}$. The observatory will be used to measure high-energy protons and cosmic rays via detection of the energetic secondary particles reaching the ground when one of these particles interacts in the atmosphere above the detector. HAWC is under construction at a site 4100 meters above sea level on the northern slope of the volcano Sierra Negra, which is located in central Mexico at $19^{\circ} \mathrm{N}$ latitude. It is scheduled for completion in 2014. In this paper we estimate the sensitivity of the HAWC instrument to point-like and extended sources of gamma rays. The source fluxes are modeled using both unbroken power laws and power laws with exponential cutoffs. HAWC, in one year, is sensitive to point sources with integral powerlaw spectra as low as $5 \times 10^{-13} \mathrm{~cm}^{-2} \mathrm{sec}^{-1}$ above $2 \mathrm{TeV}$ (approximately 50 mCrab) over $5 \mathrm{sr}$ of the sky. This is a conservative estimate based on simple event parameters and is expected to improve as the data analysis techniques are refined. We discuss known $\mathrm{TeV}$ sources and the scientific contributions that HAWC can make to our understanding of particle acceleration in these sources.
\end{abstract}

\title{
1. Introduction
}

$\mathrm{TeV}$ astronomy began in 1989 when gamma rays from the Crab Nebula were recorded at the Whipple Observatory during 81 hours of observation [1]. Due to the very low flux of $\mathrm{TeV}$ gamma rays at Earth (about $10^{-11}$ photons $\mathrm{cm}^{-2}$ $\mathrm{s}^{-1} \mathrm{TeV}^{-1}$ at $1 \mathrm{TeV}$ ) the Whipple telescope observed gamma rays indirectly by imaging the Cherenkov light produced in the atmosphere by gamma-ray extensive air showers. Since 1989, the number of confirmed TeV sources has grown to about 140 objects [2], with most of the observations made by Imaging Atmospheric Cherenkov Telescopes (IACTs) based on the Whipple design. The 
current generation of IACTs - HESS [3], VERITAS [4], and MAGIC [5] - are sensitive enough to measure the Crab Nebula at the $5 \sigma$ level with only several minutes of observations.

A second method of observing $\mathrm{TeV}$ gamma rays, known as the water Cherenkov technique, was developed at the Milagro Observatory [6], which operated from 2000 to 2008 in the mountains above Los Alamos, NM. Rather than image the Cherenkov light from extensive air showers, the Milagro detector was designed to directly sample shower particles at ground level using a large pool of water in an optically isolated reservoir. The reservoir was instrumented with an array of photomultiplier tubes (PMTs) to record the Cherenkov light produced by charged particles entering the water.

For each air shower observed in the array, the timing and amplitude of signals in the PMTs can be used to estimate the arrival direction of the primary particle and the position of the shower core. With this technique it is also possible to discriminate showers produced by photons from air showers produced by cosmic-ray hadrons, because hadronic showers characteristically produce isolated regions of high energy deposition far from the shower core. While the Milagro detector was less sensitive to point sources than the current generation of IACTs, it had the advantages of $>90 \%$ uptime and a large instantaneous field of view. As a result, the experiment was used to discover new Galactic sources of $\mathrm{TeV}$ gamma rays 7], diffuse emission from the Galactic plane [8] [9], and measure an anisotropy in the arrival direction distribution of charged cosmic rays [10] [11].

Following the success of Milagro, the High Altitude Water Cherenkov detector (HAWC) is a water Cherenkov extensive air shower array under construction at Sierra Negra, Mexico. HAWC will consist of an array of 300 water Cherenkov detectors (WCDs). Each WCD comprises a steel tank $7.3 \mathrm{~m}$ in diameter and $4.5 \mathrm{~m}$ in height, a plastic bladder to contain 200000 liters of purified water, and four PMTs: three 8-inch Hamamatsu R5912 PMTs re-used from Milagro and one 10-inch R7081-MOD high-quantum efficiency PMT. The WCDs will be deployed in a close-packed array over an area of approximately $20000 \mathrm{~m}^{2}$. The PMTs detect Cherenkov light from energetic particles passing through the WCDs. The air shower core is determined by a fit to the amount of light detected by each PMT and the direction of the incident particle is fit by the time (measured to $\sim 2 \mathrm{~ns}$ ) each PMT is hit.

The HAWC instrument, the reconstruction and analysis algorithms, and the sensitivity to gamma-ray bursts, focusing on emission below $1 \mathrm{TeV}$, have been described previously [12]. In the following sections we present the results of a study of the sensitivity of HAWC to steady sources of gamma rays from $1 \mathrm{TeV}$ to $100 \mathrm{TeV}$.

\section{Analysis Technique and Cuts}

When observing gamma-ray sources in the $100 \mathrm{GeV}-100 \mathrm{TeV}$ range, the dominant background comes from the abundant population of hadronic cosmic 
rays, mostly protons and helium nuclei. These cosmic rays arrive nearly isotropically, with anisotropy present at a $10^{-3}$ level [10] 11]. A source of gamma rays appears as a small localized bump on top of this smooth background. The analysis of a potential source consists of defining a small angular bin around the position of the source, determining the number and statistical uncertainty of background events that will appear in this angular bin, and measuring the number of events above background in the bin. We attempt to remove hadronic events with a set of cuts intended to identify the penetrating particles (mostly muons) characteristic of a hadronic air shower.

Simulations are used to model the air shower and ground detector components. The air shower is modeled using CORSIKA 13] and the detector components are modeled with Geant4 [14]. The reconstruction and gamma/hadron separation algorithms are applied to the simulated output. The calculation of sensitivity to high-energy steady sources is similar to the analysis described in 12]. Since the publication of a previous study 12], the design of HAWC has been modified to include an additional 10-inch high-efficiency PMT at the center of each WCD. The current sensitivity computation includes this PMT modeled as one of the re-used Milagro 8-inch PMTs. This provides a conservative estimate to the sensitivity while deferring the issue of developing appropriate algorithms to handle the two distinct types of PMTs.

We use the core and angular reconstruction algorithms and calculate a "compactness" parameter for photon/hadron discrimination as in 12. The compactness parameter is designed to identify muons in the air shower, which appear as localized charge depositions. The compactness is defined as $\mathrm{N}_{\text {chan }} / \mathrm{CxPE}_{40}$ where $\mathrm{N}_{\text {chan }}$ is the number of PMTs participating in an event and $\mathrm{CxPE}_{40}$ is the total number of photo-electrons (PEs) in the PMT with the largest signal that is located outside a radius of 40 meters from the reconstructed air shower core. However, the optimization of the cuts for the present sensitivity calculation differs from [12] because we are primarily interested in high-energy sources with spectra extending beyond $1 \mathrm{TeV}$.

The performance of the HAWC detector, notably the angular resolution and gamma/hadron separation, improves with the size of the air shower on the ground. Larger air showers are better measured and easier to reconstruct. Furthermore, the number of muons in a cosmic ray air shower increases with the energy of the incident cosmic ray, and so the gamma/hadron discrimination also improves with energy. After hadron removal cuts and a cut on the angular distance of events to a source, we anticipate (for a Crab-like point source source) a signal-to-background ratio of between 1:350 at $100 \mathrm{GeV}$ to about 10:1, at 10 $\mathrm{TeV}$. The event parameters used here are relatively simple. We anticipate the sensitivity to improve beyond what is presented here as the event reconstruction is improved.

In order to account for the energy dependence of the sensitivity, the data are divided into 14 bins using $\mathrm{N}_{\text {chan }}$, the total number of PMTs hit during an event, and $\mathrm{N}_{\mathrm{PE}}$, the total number of photo-electrons (PEs) measured by the PMTs during the event. This binning is somewhat arbitrary but its selection does not impact the sensitivity appreciably. Both $\mathrm{N}_{\text {chan }}$ and $\mathrm{N}_{\mathrm{PE}}$ are corre- 
lated with shower energy, but neither is an ideal energy estimator because they do not account for the atmospheric slant path of the shower geometry, nor do they account for how well-contained ths shower is within the array. More sophisticated energy estimation algorithms are expected in the future. The bins are arranged so that they reflect larger - and thus higher-energy - events.

The 14 bins chosen for $\mathrm{N}_{\text {chan }}$ and $\mathrm{N}_{\mathrm{PE}}$ are shown in Table 1. Bins 0-8 are determined using $\mathrm{N}_{\text {chan }}$. Higher bins are determined by $\mathrm{N}_{\mathrm{PE}}$ because $\mathrm{N}_{\text {chan }}$ saturates as an energy estimator when $\sim 3 / 4$ of the array is hit For each bin, we determine the angular bin and compactness cut that maximizes the statistical significance of that bin. The optimization is made by maximizing the statistical significance of a hypothetical source with a differential photon flux [3] of

$$
d N / d E=3.45 \times 10^{-11}(E / \mathrm{TeV})^{-2.63} \mathrm{~cm}^{-2} \mathrm{~s}^{-1} \mathrm{TeV}^{-1}
$$

and a declination of $+35^{\circ}$. The declination $+35^{\circ}$ is chosen because it is characteristic of sources in the field-of-view of HAWC, which is located at $19^{\circ} \mathrm{N}$. The spectral index $\alpha=-2.63$ has been chosen as a reasonable compromise between sources with photon fluxes concentrated at the low and high-energy limits of the HAWC energy range.

To calculate the significance of a point source, we assume that we are measuring the source at a fixed declination over many transits of the source. We weight the simulated events by the amount of time spent at each zenith in order to account for the source transit. We then calculate the number of expected signal and background events in our analysis bins. Table 1 shows the results of this optimization.

For a given hypothetical source, we wish to compute the significance of one year of observations while accounting for the changing signal and background efficiencies in each bin. To do so, we assign a weight $w_{i}=S_{i}^{\mathrm{opt}} / B_{i}^{\mathrm{opt}}$ to each analysis bin $i$, where $S_{i}^{\text {opt }}$ and $B_{i}^{\text {opt }}$ are the expected signal and background from the optimization hypothesis. If the expected signal and background counts from a given source are $S_{i}$ and $B_{i}$, which could in general be different than for the $S_{i}^{\text {opt }}$ and $B_{i}^{\text {opt }}$ of the optimization hypothesis, then we calculate the significance of the observation as

$$
\sigma=\frac{\sum_{i} w_{i} \cdot S_{i}}{\sqrt{\sum_{i} w_{i}^{2} B_{i}}}
$$

where the sum is taken over the 14 analysis bins.

Figure 1 shows the performance of the angular localization and photon discrimination after applying the optimized cuts from Table 1. As one can see, the angular resolution improves steadily with increasing energy, reaching $\sim 0.1^{\circ}$ at $\mathrm{E}>10 \mathrm{TeV}$. The hadron rejection efficiency improves with increasing energy up to about $10 \mathrm{TeV}$, at which point none of the simulate background survive cuts. This makes the analysis difficult to optimize at high energy. As a conservative approach, we choose the cut that preserves at least 10 simulated background events to estimate the hadron rejection efficiency. This approach is likely to produce an underestimate in the predicted sensitivity at high energies. The issue will be solved when the experiment starts taking data because the data 


\begin{tabular}{|c|c|c|c|c|c|c|c|c|}
\hline Bin & $\begin{array}{l}\mathrm{N}_{\text {chan }} \\
\text { Bin }\end{array}$ & $\begin{array}{c}\log _{10}\left(N_{P E}\right) \\
\text { Bin }\end{array}$ & $\begin{array}{c}\text { Angular } \\
\text { Bin } \\
\text { (deg.) }\end{array}$ & $\begin{array}{c}\text { Compact. } \\
\text { Cut } \\
\left(\mathrm{PE}^{-1}\right)\end{array}$ & $\begin{array}{c}\text { Signal } \\
S_{i}\end{array}$ & $\begin{array}{c}\text { Weight } \\
w_{i}\end{array}$ & $\begin{array}{c}E_{\log } \\
(\mathrm{GeV})\end{array}$ & $\sigma_{E_{\log }}$ \\
\hline 1 & $\begin{array}{l}39-59 \\
\end{array}$ & $1.0-7.0$ & 1.30 & 3.1 & $5.7 \times 10^{4}$ & $2.8 \times 10^{-3}$ & 2.5 & 0.46 \\
\hline 2 & $60-69$ & $1.0-7.0$ & 1.00 & 5.2 & $1.4 \times 10^{4}$ & $9.6 \times 10^{-3}$ & 2.6 & 0.47 \\
\hline 3 & $70-90$ & $1.0-7.0$ & 0.88 & 5.3 & $1.8 \times 10^{4}$ & $1.3 \times 10^{-2}$ & 2.7 & 0.44 \\
\hline 4 & $91-147$ & $1.0-7.0$ & 0.68 & 8.1 & $1.8 \times 10^{4}$ & $4.4 \times 10^{-2}$ & 2.9 & 0.40 \\
\hline 5 & $148-231$ & $1.0-7.0$ & 0.50 & 11.7 & $7.9 \times 10^{3}$ & $1.7 \times 10^{-1}$ & 3.0 & 0.35 \\
\hline 6 & $232-349$ & $1.0-7.0$ & 0.36 & 13.4 & $3.7 \times 10^{3}$ & $4.9 \times 10^{-1}$ & 3.2 & 0.32 \\
\hline 7 & $350-495$ & $1.0-7.0$ & 0.30 & 17.2 & $1.4 \times 10^{3}$ & 2.0 & 3.5 & 0.28 \\
\hline 8 & $496-655$ & $1.0-7.0$ & 0.22 & 17.7 & $6.0 \times 10^{2}$ & 4.7 & 3.7 & 0.24 \\
\hline 9 & 656-789 & $1.0-7.0$ & 0.20 & 17.1 & $2.4 \times 10^{2}$ & 9.8 & 3.8 & 0.21 \\
\hline 10 & $790-1200$ & $4.0-4.5$ & 0.16 & 14.4 & $1.4 \times 10^{2}$ & $1.6 \times 10^{1}$ & 4.0 & 0.18 \\
\hline 11 & $790-1200$ & $4.5-4.9$ & 0.14 & 11.5 & $1.2 \times 10^{2}$ & $1.2 \times 10^{1}$ & 4.2 & 0.18 \\
\hline 12 & $790-1200$ & $4.9-5.3$ & 0.12 & 7.2 & $2.5 \times 10^{1}$ & $1.2 \times 10^{1}$ & 4.6 & 0.07 \\
\hline 13 & $790-1200$ & $5.3-5.7$ & 0.12 & 1.9 & 3.4 & $1.8 \times 10^{-1}$ & 5.1 & 0.13 \\
\hline 14 & $790-1200$ & $5.7-6.4$ & 0.08 & 0.9 & $3.2 \times 10^{-1}$ & $3.6 \times 10^{-1}$ & 5.5 & 0.10 \\
\hline
\end{tabular}

Table 1: The table shows the $\mathrm{N}_{\text {chan }}$ and $\log _{10}\left(\mathrm{~N}_{\mathrm{PE}}\right)$ values used to define the analysis bins along with angular bin and compactness cut chosen to optimize the statistical significance of the hypothetical source in Equation 1 at $+35^{\circ}$ declination. Also shown are the number of photon events from our hypothetical source passing cuts, and the weights used in Equation 2 optimized for the hypothetical photon flux given in Equation 1. Also shown are the median and width, in logarithmic space, of the true photon energy distribution of events $E_{\log }=\log _{10}(E / \mathrm{GeV})$ in each bin. The results are presented for one year of data taking. 

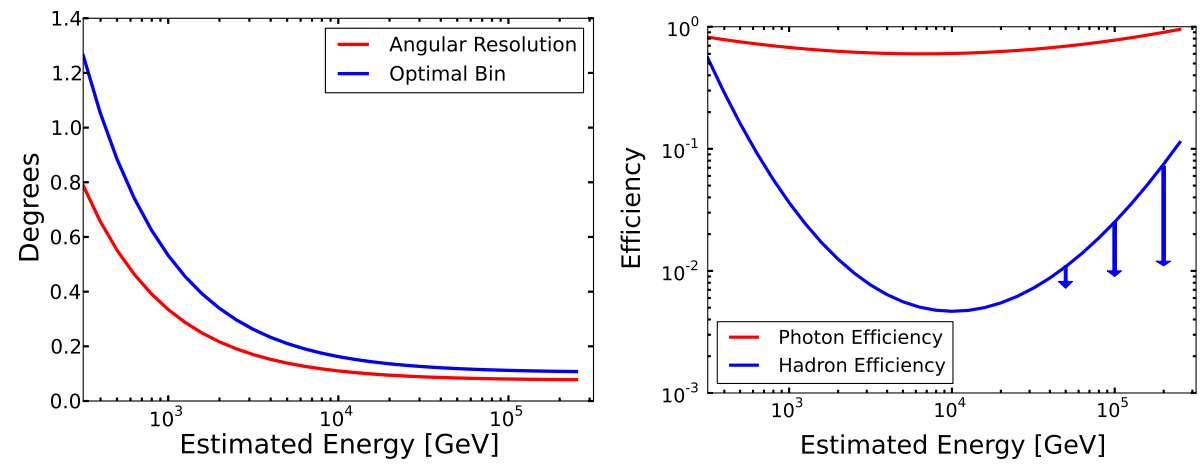

Figure 1: Performance of the optimal cuts from Table 1 The left figure shows the angular resolution (one standard deviation of a fitted two-dimensional Gaussian) and optimal angular bin (column 4) for the energy bins from Table 1. smoothed for display. The figure on the right shows the efficiency of the compactness cut, after all other cuts have been made, on the photon signal and background in the bins from Table 1 . Beyond $\sim 10 \mathrm{TeV}$ the photon discrimination becomes strong enough to remove all of the existing simulated background events, and we conservatively require 10 simulated events to survive cuts so the background can be reliably estimated. The arrows above $10 \mathrm{TeV}$ emphasize that the hadron rejection is anticipated to improve.

(dominated by hadronic background) can be used to estimate the rejection directly. Nevertheless the HAWC sensitivity above $10 \mathrm{TeV}$ is very close to the limit implied by the need to detect at least $\sim 10$ events.

Since many Galactic sources are extended, we also consider the sensitivity of the instrument to sources that are extended by some amount. We presume that the spatial extent of the source is a disk with a radius $R_{S}$ at all photon energies. The measured distribution is then the convolution of the true source distribution and the response of the instrument to a point source. Once the source is smeared by the point response of the instrument, the optimal angular bin is calculated to maximize Equation 2. In general, the optimal bin size of an extended source will be larger than that of a point source, meaning more background is admitted. All other cuts remain the same.

\section{Results}

In our analyis, spectra of the form

$$
\mathrm{dN} / \mathrm{dE}=\Phi_{0}(\mathrm{E} / \mathrm{TeV})^{-\alpha} \exp \left(-\mathrm{E} / \mathrm{E}_{\text {cut }}\right)
$$

are considered, where $\Phi_{0}$ is the flux at $1 \mathrm{TeV}, \alpha$ is the source spectral index and $\mathrm{E}_{\text {cut }}$ is the energy at which the spectrum cuts off. While a source may have a more complicated energy spectrum, the power-law with an exponential cutoff has been sufficient to describe most $\mathrm{TeV}$ sources to date. 
Figure 2 shows the sensitivity of HAWC to sources of varying spectral and spatial parameters. To be considered within the sensitive range of the instrument the mean signal expectation must be detectable at 5 standard deviations (Equation 2) above background with a full year of data. Note that one year of data presumes one year's worth of transits of the source overhead and includes a substantial amount of time with the hypothetical source out of the instrument's field-of-view. The top panel of Figure 2 shows the sensitivity of the detector to sources with no cutoff $\left(E_{\text {cut }}=\infty\right)$ as a function of the source's declination. The center panel shows the sensitivity to a source at $+35^{\circ}$ declination as a function of cutoff energy assuming two different values for the differential spectral index. The bottom panel shows the sensitivity to a source at $+35^{\circ}$ declination as a function of the spatial extent of the source assuming a pure power law spectrum with no cutoff. The flux sensitivity is expressed as the integral flux above 2 $\mathrm{TeV}$. This choice nearly eliminates the dependence of the sensitivity of pure power-law spectra on the spectral index.

We find that over one year of exposure, HAWC is sensitive to pure power law spectra at a level of $5 \times 10^{-13} \mathrm{~cm}^{-2} \mathrm{sec}^{-1}(\sim 50 \mathrm{mCrab})$ above $2 \mathrm{TeV}$ over 5 sr $(40 \%)$ of the sky. Figure 3 shows the sensitivity to differential $E^{-2}$ spectra in Galactic coordinates along with sources from the TeVCat catalog [2]. It is worth noting that the form of Equation 2 indicates that if a source were to flare by a factor of $N$, the time required to see the source at fixed significance would be reduced by $N^{2}$. Similarly, since HAWC is roughly 15 times more sensitive than Milagro, we anticipate to see the Milagro sky $\sim 225$ times faster, i.e. in just over a week of data-taking with the full HAWC instrument.

The HAWC differential sensitivity is shown alongside the sensitivity from other current instruments in Figure 4 HAWC complements these instruments with its high-energy reach and its wide field-of-view. Whereas an IACT must point at a given source on dark, clear nights in order to observe it and can observe $\sim 15$ sources with 50 hours of exposure in a year, HAWC continuously records events across the entire overhead sky. This capability is useful for the recording of transient source events, and assists in the energy reach of the instrument above $1 \mathrm{TeV}$. The power-law spectrum of a typical astrophysical source means extreme high-energy events are very rare, making continuous observations important.

\section{Discussion}

The HAWC instrument is designed to study particle acceleration in Galactic and extra-Galactic sources as well as the propagation of high-energy particles through the Galaxy and the extra-Galactic background light (EBL). Table 2 illustrates the scientific potential of HAWC by projecting a number of estimated HAWC measurements on a selection of known TeV sources.

Pulsar Wind Nebulae (PWN) are the most common Galactic source of $\mathrm{TeV}$ gamma rays [26] 27]. A central rotating neutron star powers an electromagneticallydriven flow of energetic electrons: the pulsar wind. These electrons can radiate in the surround material and can be further accelerated in shocks. 

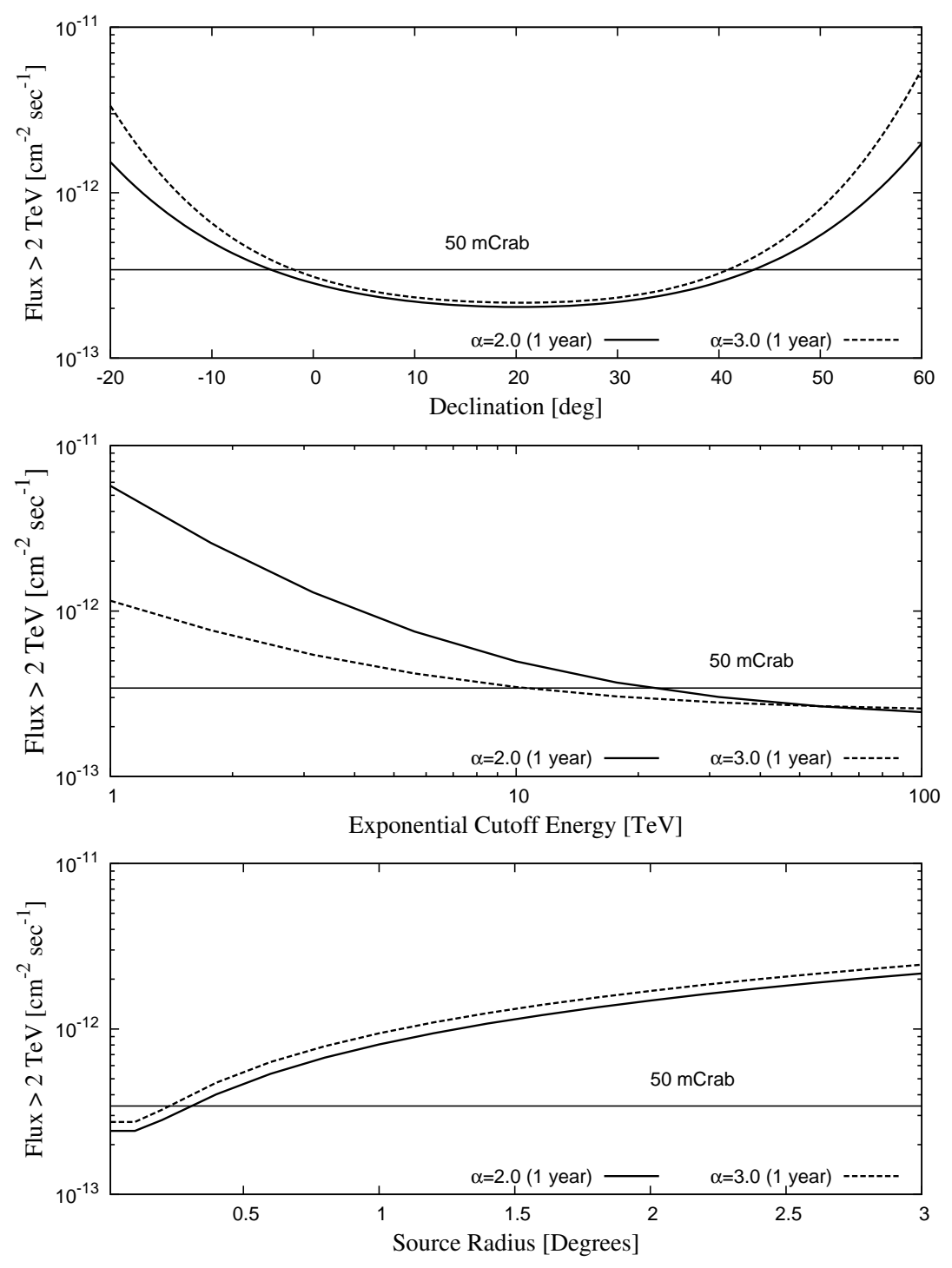

Figure 2: The sensitivity of HAWC to sources with varying spectral parameter. We show the flux required to give a central expectation of $5 \sigma$ in one year. The top panel shows the sensitivity of HAWC to sources with pure power-law spectra as a function of declination. In the center panel, the sensitivity to a source at $+35^{\circ}$ declination is shown as a function of spectral cutoff energy. In the bottom panel, the sensitivity to a source (also at $+35^{\circ}$ ) with a pure power law spectrum is shown as a function of the spatial extent of the source. Note that one day of data corresponds to one transit of the source, which means the source spends only a few hours in the field of view of the detector. For the computation of the integral Crab flux, we have assumed the pure power-law measurement from [3] which is the same as is given in Equation 1 . 


\begin{tabular}{|c|c|c|c|c|c|c|c|}
\hline Name & $\begin{array}{c}\text { RA } \\
(\text { deg })\end{array}$ & $\begin{array}{l}\text { Dec } \\
(\operatorname{deg})\end{array}$ & $\alpha$ & $\begin{array}{l}E_{c u t} \\
(\mathrm{TeV})\end{array}$ & $\begin{array}{c}\text { Flux }(>2 \mathrm{TeV}) \\
1 \times 10^{13} \text { photon } /\left(\mathrm{cm}^{2} \mathrm{~s}\right)\end{array}$ & Significance & Reference \\
\hline Crab (Milagro) & 83.6 & 22.0 & 3.1 & $\infty$ & 91.1 & 229.9 & [6] \\
\hline Crab (Milagro) & & & 2.6 & 31 & 79.2 & 163.1 & [6] \\
\hline Crab (Reference) & & & 2.63 & $\infty$ & 68.5 & 147.8 & [3] \\
\hline MGRO J2019+37 & 206.1 & 18.4 & 2.8 & $\infty$ & 40.5 & 66.8 & [21] \\
\hline MGRO J1908+06 & 287.1 & 6.18 & 2.1 & $\infty$ & 17.6 & 36.6 & {$[22]$} \\
\hline MGRO J2031+41 & 308.0 & 41.6 & 3.2 & $\infty$ & 33.8 & 53.0 & [21] \\
\hline MRK 421 (very low) & 166.1 & 38.2 & 2.29 & 1.59 & 18.4 & 31.5 & [23] \\
\hline MRK 421 (mid) & & & 2.28 & 4.36 & 131.5 & 178.0 & [23] \\
\hline MRK 421 (very high) & & & 1.87 & 2.74 & 462.9 & 567.9 & [23] \\
\hline M87 & 187.7 & 12.2 & 2.31 & $\infty$ & 2.3 & 4.7 & [24] \\
\hline IC443 (hard) & 94.2 & 22.5 & 2.61 & $\infty$ & 2.6 & 5.6 & $25]$ \\
\hline IC443 (measured) & & & 2.99 & $\infty$ & 1.1 & 2.5 & {$[25]$} \\
\hline IC443 (soft) & & & 3.37 & $\infty$ & 0.4 & 1.3 & [25] \\
\hline
\end{tabular}

Table 2: Table of a selection of known $\mathrm{TeV}$ sources (not complete) shown with the expected measurement significance after one year of HAWC data, assuming the sources are point sources, under specific hypotheses for the energy spectrum. 


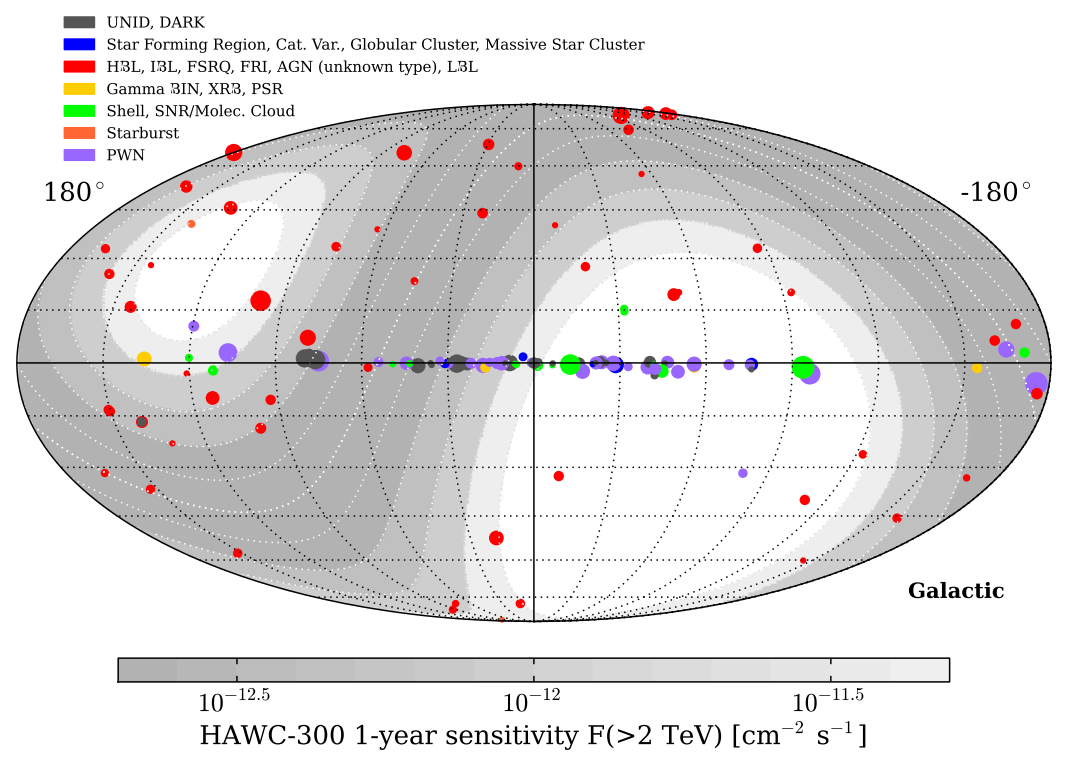

Figure 3: The sensitivity of HAWC to differential $E^{-2}$ spectra along with known sources from the TeVCat TeV catalog [2] in Galactic coordinates. The size of the source is representative of the source's flux in the TeVCat catalog.

In a surprising twist to conventional thinking on PWN, recent flares of the Crab Nebula have been observed at energies between $100 \mathrm{MeV}$ and $100 \mathrm{GeV}$ by AGILE [28] and the Fermi-LAT [29]. Above $1 \mathrm{TeV}$, the ARGO-YBJ collaboration has announced possible evidence for variability [30] though variability has not been yet observed by IACT experiments. The Fermi data show strong transient flares in the synchrotron emission from the Crab Nebula, peaking at 50 times the quiescent state, over several days.

To account for the rapid flaring, it has been suggested that the flares may be due to acceleration by magnetic reconnection rather than shock acceleration (e.g. 31]). Currently, we do not know if the flares are confined to the synchrotron emission or whether they extend to the higher-energy inverse-Compton emission. HAWC will be able to detect an order-of-magnitude flare of the Crab in less than 10 minutes and will identify or constrain the inverse-Compton emission from these flares. Furthermore, it is currently not known whether other PWN flare like the Crab. HAWC will continuously monitor other PWN for flares.

Supernova Remnants (SNR) 32] have been observed to emit multi-TeV photons. SNRs are the leading candidates for cosmic ray acceleration in the Galaxy, and $\mathrm{TeV}$ emission can provide indirect evidence of hadronic acceleration inside these objects. Compelling evidence of cosmic ray acceleration comes from the association of $\mathrm{TeV}$ photons from SNR interacting with nearby molecular clouds, which would be inconsistent with $\mathrm{TeV}$ emission from inverse Compton scattering. As a specific example, the $\mathrm{TeV}$ emission from the SNR IC 443 measured 


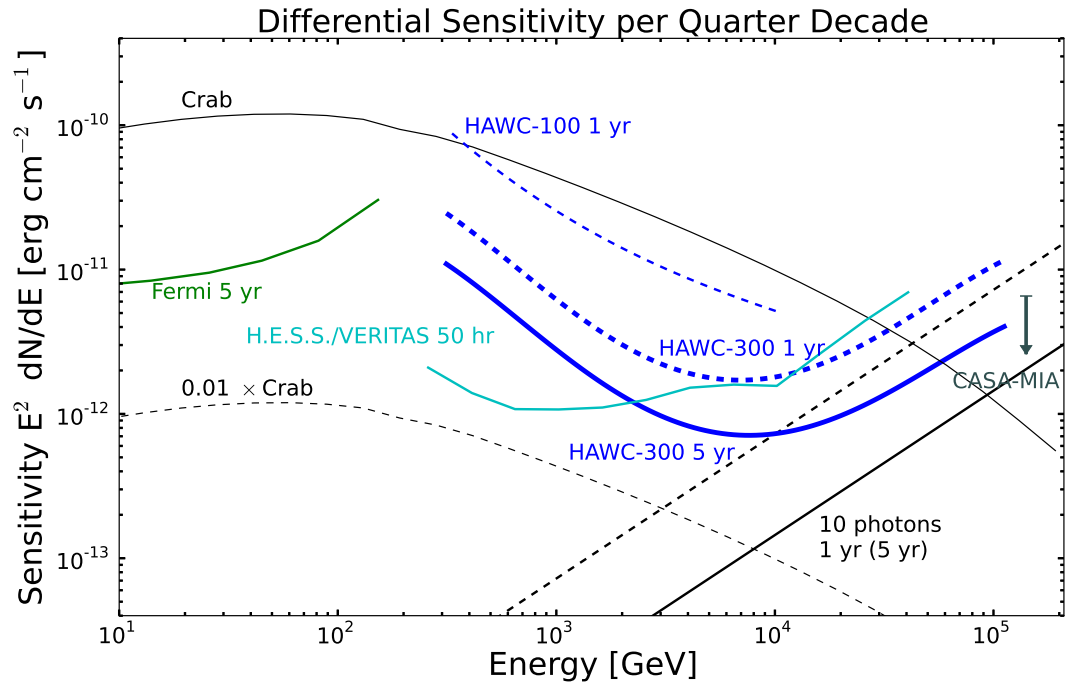

Figure 4: The differential sensitivity of HAWC compared to peer instruments. The sensitivity is found by calculating the flux needed for a $5 \sigma$ detection for quarter-decade energy bins using Eqn. 17 from [15]. Quarter-decade energy bins are found by normalizing the bins in Table 1 to facilitate comparison to 50-hour IACT [3] 16] 17] and 3-year Fermi 18] sensitivity calculations. Note that the actual triggering threshold extends somewhat lower than implied by this figure, down to $100 \mathrm{GeV}$. We show the flux required to produce 10 events in $20000 \mathrm{~m}^{2}$ assuming a 5 hour observation each day and $40 \%$ loss of efficiency due to angular bin cuts and a $50 \%$ loss of efficiency due to photon/hadron discrimination. Without improvement to the high-energy effective area, this is a practical limit to the HAWC sensitivity. Also shown, for reference, is the measured Crab flux from [19] and an inferred quarter-decade differential upper limit from the Crab at $141 \mathrm{TeV}$ from the CASA-MIA experiment [20]. The sensitivity for the first 100 of the 300 HAWC WCDs is shown for comparison. 
by VERITAS 25] has been interpreted as the interaction of cosmic rays inside a molecular cloud. This interpretation was strengthened by the observation of the characteristic neutral pion decay spectrum by Fermi 33]. The measured spectral index of IC 443 above $1 \mathrm{TeV}$ is relatively uncertain but it will be detectable with HAWC after only a year of data-taking if the spectrum is as hard as the VERITAS error bars allow. Particularly intriguing for IC 443 is the Milagro $3 \sigma$ excess at $35 \mathrm{TeV}$ [34], which, if confirmed, is 10 times larger than anticipated from extrapolating the central values of the VERITAS measurement. Given the uncertainty in the spectral index measured by VERITAS and the Milagro point, it may be that the spectrum from IC 443 is in fact harder than measured or that a second hard component is measured by Milagro. HAWC will resolve this question for IC 443 and other SNR.

HAWC will study the TeV emission from Active Galactic Nuclei (AGN). AGN emission can be extremely variable with flares up to ten times the quiescent flux or more (e.g. 23] ). The large distances to many AGN mean that HAWC data can be used to study the far-infrared component of the EBL 35], secondary gamma-ray production between cosmic-ray sources and Earth 36], and even exotic modes of inter-galactic particle transport such as axions 37]. Furthermore, HAWC will provide unparalleled sensitivity to TeV AGN flares across the entire overhead sky. This is particularly interesting given the occurrence of so-called "orphan" flares in which the inverse-Compton emission from a blazar will flare without an accompanying flare in the synchrotron emission 38]. It has been suggested that these flares, defying explanation by the simplest Synchrotron Self-Compton models, may indicate the presence of hadronic acceleration in the blazar [39] [40]. With IACTs blind to most of the overhead sky, and with no corresponding synchrotron flare to trigger a search, an instrument like HAWC is needed to observe these flares. Identified orphan flares can be used to trigger searches in neutrino telescopes such as IceCube [4]. Detected contemporaneous neutrino emission would provide concrete evidence of hadron acceleration in blazars.

HAWC data will also be used to study physics beyond the Standard Model. For example, searches for gamma-ray emission from high-mass, low-luminosity satellites of the Milky Way are a useful channel for detecting dark matter particles with masses over $1 \mathrm{TeV}$ [42] 43] 44]. If dark matter interactions are occurring in such objects, these processes will likely result in the production of secondary gamma rays. The production of gamma rays from cosmic-ray acceleration and interaction in these objects is expected to be very low, so an observation of gamma rays may indicate the presence of dark matter interactions. Typical photon energy spectra from the annihilation or decay of dark matter of mass $m_{\chi}$ are concentrated at photon energies about a factor of 10 lower than $m_{\chi}$. The superior sensitivity of HAWC above a photon energy of $1 \mathrm{TeV}$ implies that HAWC will provide competitive limits on dark matter annihilation and decay for $m_{\chi}$ above $\sim 10 \mathrm{TeV}$, depending on the annihilation or decay channel. 


\section{Conclusion}

Multi-TeV gamma rays are a probe of particle acceleration in astrophysical sources. These sources can be transient, flaring by an order of magnitude or more in a matter of hours, with spectra already measured to $20 \mathrm{TeV}$ or more with current instruments. The High Altitude Water Cherenkov observatory is sensitive to photons from $100 \mathrm{GeV}$ to $100 \mathrm{TeV}$, with a peak sensitivity at the 10-20 TeV, where existing IACT spectra end. HAWC will observe the entire overhead sky for transient emission and extend and constrain spectra up to 100 $\mathrm{TeV}$. HAWC will survey the sky with a point-source sensitivity of $5 \times 10^{-13}$ $\mathrm{cm}^{-2} \mathrm{sec}^{-1}$ above $2 \mathrm{TeV}$ in a year of data-taking across $5 \mathrm{sr}(40 \%)$ of the sky. When completed, HAWC will be the most sensitive gamma-ray detector above $10 \mathrm{TeV}$ and will maintain unprecedented wide-field sensitivity to gamma rays above $100 \mathrm{GeV}$.

\section{Acknowledgments}

We gratefully acknowledge Scott DeLay his dedicated efforts in the construction and maintenance of the HAWC experiment. This work has been supported by: the National Science Foundation, the US Department of Energy Office of High-Energy Physics, the LDRD program of Los Alamos National Laboratory, Consejo Nacional de Ciencia y Tecnologia (grants 55155, 103520, 105033, 105666, 122331 and 132197), Red de Fisica de Altas Energias, DGAPA-UNAM (grants IN105211, IN112910 and IN121309, IN115409), VIEP-BUAP (grant 161-

EXC-2011), the University of Wisconsin Alumni Research Foundation, and the Institute of Geophysics and Planetary Physics at Los Alamos National Lab.

\section{References}

[1] T. C. Weekes et al. Observation of $\mathrm{TeV}$ gamma rays from the Crab nebula using the atmospheric Cerenkov imaging technique. ApJ, 342:379-395, July 1989.

[2] S. Wakely and D. Horan. http://tevcat.uchicago.edu/.

[3] F. Aharonian et al. Observations of the Crab nebula with HESS. A\&A, 457:899-915, October 2006.

[4] O. Celik. Observations of the Crab Nebula and Pulsar with VERITAS. In International Cosmic Ray Conference, volume 2 of International Cosmic Ray Conference, pages 847-850, 2008.

[5] E. Aliu et al. Observation of Pulsed $\gamma$-Rays Above $25 \mathrm{GeV}$ from the Crab Pulsar with MAGIC. Science, 322:1221-, November 2008.

[6] A. A. Abdo et al. Observation and Spectral Measurements of the Crab Nebula with Milagro. ApJ, 750:63, May 2012. 
[7] A. A. Abdo et al. TeV Gamma-Ray Sources from a Survey of the Galactic Plane with Milagro. ApJL, 664:L91-L94, August 2007.

[8] A. A. Abdo et al. A Measurement of the Spatial Distribution of Diffuse TeV Gamma-Ray Emission from the Galactic Plane with Milagro. ApJ, 688:1078-1083, December 2008.

[9] A. A. Abdo et al. Discovery of TeV Gamma-Ray Emission from the Cygnus Region of the Galaxy. ApJL, 658:L33-L36, March 2007.

[10] A. A. Abdo et al. Discovery of Localized Regions of Excess 10-TeV Cosmic Rays. Physical Review Letters, 101(22):221101, November 2008.

[11] A. A. Abdo et al. The Large-Scale Cosmic-Ray Anisotropy as Observed with Milagro. ApJ, 698:2121-2130, June 2009.

[12] A. U. Abeysekara et al. On the sensitivity of the HAWC observatory to gamma-ray bursts. Astroparticle Physics, 35:641-650, May 2012.

[13] D. Heck, J. Knapp, J. N. Capdevielle, G. Schatz, and T. Thouw. CORSIKA: a Monte Carlo code to simulate extensive air showers. February 1998.

[14] S. Agostinelli et al. Geant4 - a simulation toolkit. Nuclear Instruments and Methods in Physics Research Section A: Accelerators, Spectrometers, Detectors and Associated Equipment, 506(3):250 - 303, 2003.

[15] T.-P. Li and Y.-Q. Ma. Analysis methods for results in gamma-ray astronomy. ApJ, 272:317-324, September 1983.

[16] J. Aleksić et al. Performance of the MAGIC stereo system obtained with Crab Nebula data. Astroparticle Physics, 35:435-448, February 2012.

[17] J. Holder. private communication, 2013.

[18] M. Ackermann et al. The Fermi Large Area Telescope on Orbit: Event Classification, Instrument Response Functions, and Calibration. ApJS, 203:4, November 2012.

[19] F. Aharonian et al. The Crab Nebula and Pulsar between $500 \mathrm{GeV}$ and 80 TeV: Observations with the HEGRA Stereoscopic Air Cerenkov Telescopes. ApJ, 614:897-913, October 2004.

[20] A. Borione et al. A Search for Ultra-High-Energy Gamma-Ray Emission from the Crab Nebula and Pulsar. ApJ, 481:313, May 1997.

[21] A. A. Abdo et al. Spectrum and Morphology of the Two Brightest Milagro Sources in the Cygnus Region: MGRO J2019+37 and MGRO J2031+41. ApJ, 753:159, July 2012. 
[22] A. Djannati-Atai et al. H.E.S.S. Galactic Plane Survey unveils a Milagro Hotspot. In Proc. 30th International Cosmic Ray Conference, Merida, volume 2, pages 831-834, December 2007.

[23] V. A. Acciari et al. TeV and Multi-wavelength Observations of Mrk 421 in 2006-2008. ApJ, 738:25, September 2011.

[24] V. A. Acciari et al. Observation of Gamma-Ray Emission from the Galaxy M87 above $250 \mathrm{GeV}$ with VERITAS. ApJ, 679:397-403, May 2008.

[25] T. B. Humensky et al. VERITAS Studies of the Supernova Remnants Cas A and IC 443. In American Institute of Physics Conference Series, volume 1085, pages 357-360, December 2008.

[26] B. M. Gaensler and P. O. Slane. The Evolution and Structure of Pulsar Wind Nebulae. ARA\&A, 44:17-47, September 2006.

[27] S. P. Reynolds, B. M. Gaensler, and F. Bocchino. Magnetic Fields in Supernova Remnants and Pulsar-Wind Nebulae. Space Sci. Rev., 166:231261, May 2012.

[28] M. Tavani et al. Discovery of Powerful Gamma-Ray Flares from the Crab Nebula. Science, 331:736-, February 2011.

[29] A. A. Abdo et al. Gamma-Ray Flares from the Crab Nebula. Science, 331:739-, February 2011.

[30] G. Aielli et al. Enhanced $\mathrm{TeV}$ gamma ray flux from the Crab Nebula observed. ATel, 2921, 2010.

[31] B. Cerutti, D. A. Uzdensky, and M. C. Begelman. Extreme Particle Acceleration in Magnetic Reconnection Layers: Application to the Gamma-Ray Flares in the Crab Nebula. ApJ, 746:148, February 2012.

[32] S. P. Reynolds. Supernova Remnants at High Energy. ARA\&A, 46:89-126, September 2008.

[33] M. Ackermann et al. Detection of the Characteristic Pion-Decay Signature in Supernova Remnants. Science, 339:807-811, February 2013.

[34] A. A. Abdo et al. Milagro Observations of Multi-TeV Emission from Galactic Sources in the Fermi Bright Source List. ApJ, 700:L127-L131, August 2009.

[35] J. D. Finke, S. Razzaque, and C. D. Dermer. Modeling the Extragalactic Background Light from Stars and Dust. ApJ, 712:238-249, March 2010.

[36] W. Essey, O. E. Kalashev, A. Kusenko, and J. F. Beacom. Secondary Photons and Neutrinos from Cosmic Rays Produced by Distant Blazars. Physical Review Letters, 104(14):141102, April 2010. 
[37] D. Horns et al. Hardening of TeV gamma spectrum of AGNs in galaxy clusters by conversions of photons into axion-like particles. ArXiv e-prints, July 2012.

[38] H. Krawczynski et al. Multiwavelength Observations of Strong Flares from the TeV Blazar 1ES 1959+650. ApJ, 601:151-164, January 2004.

[39] F. Halzen and D. Hooper. High energy neutrinos from the TeV Blazar 1ES $1959+650$. Astroparticle Physics, 23:537-542, July 2005.

[40] A. Reimer, M. Böttcher, and S. Postnikov. Neutrino Emission in the Hadronic Synchrotron Mirror Model: The "Orphan" TeV Flare from 1ES 1959+650. ApJ, 630:186-190, September 2005.

[41] R. Abbasi et al. Time-dependent Searches for Point Sources of Neutrinos with the 40-string and 22-string Configurations of IceCube. ApJ, 744:1, January 2012.

[42] P. Scott et al. Direct constraints on minimal supersymmetry from Fermi-LAT observations of the dwarf galaxy Segue 1. J. Cosmology Astropart. Phys., 1:31, January 2010.

[43] E. Aliu et al. VERITAS deep observations of the dwarf spheroidal galaxy Segue 1. Phys. Rev. D, 85(6):062001, March 2012.

[44] J. Aleksic. Segue 1: the best dark matter candidate dwarf galaxy surveyed by MAGIC. In International Cosmic Ray Conference, volume 5 of International Cosmic Ray Conference, page 149, 2011. 